\title{
Co-operative Lane-Level Positioning Using Markov Localization
}

\author{
Thanh-Son Dao, Keith Yu Kit Leung, Christopher M. Clark, and Jan P. Huissoon
}

\begin{abstract}
The majority of today's navigation techniques for intelligent transportation systems use Global Positioning Systems (GPS) that can provide position information with bounded errors. However, because of the low accuracy and multi-path problem, it is challenging to determine a vehicle's position at lane level. With Markov-based approach based on sharing information among a group of vehicles that are traveling close to each other, the lane positions of vehicles can be found. The algorithm shows its effectiveness in both simulations and experiments with real data.
\end{abstract}

Index Terms - lane-level positioning, lane determination, Markov localization, GPS, vehicle navigation

\section{INTRODUCTION}

$I_{d}$ NTELLIGENT transportation systems are being developed in many different countries with an aim to improve the road traffic efficiency and safety. Many systems have been proposed and some lane finding/detection systems have been already commercialized. With the capability of lane-level positioning, a large number of transportation research applications would benefit. For example, in terms of lane departure warning/lane keeping, a safety application tracks a car's current offset from the road/lane centerline. If it deviates more than a certain amount, a warning signal could activate or the car assumes control to avoid an accident. Another application of lane-level positioning are lane-level navigation systems which advise the driver as to which specific lane he should choose to reach his destination without excess the last-minute lane changing. Or using probe vehicles, transportation officials and researchers could determine differences in traffic conditions for different lanes of the freeway. This is particularly important when understanding the efficiency of weaving sections that may unnecessarily cause recurrent congestion [1].

Due to the importance of such applications, much effort has been put in lane finding/positioning by a number of researchers. Ieng et al. [2] deal with the multi-lane detection by using multiple cameras. Pierre-Yves et al. [3] use lane-

Manuscript received March 1, 2006. This work is funded by AUTO21.

Thanh-Son Dao is a PhD candidate at the Lab for Autonomous and Intelligent Robotics, Dept. of Mechanical Engineering, University of Waterloo, ON, Canada N2L 3G1 (e-mail: tsdao@engmail.uwaterloo.ca).

Keith Yu Kit Leung is a MASc candidate at the Lab for Autonomous and Intelligent Robotics, Dept. of Mechanical Engineering, University of Waterloo, ON, Canada N2L 3G1 (e-mail: kykleung@lair.uwaterloo.ca).

Christopher M. Clark is an Assistant Professor at the Lab for Autonomous and Intelligent Robotics, Dept. of Mechanical Engineering, University of Waterloo, ON, Canada N2L 3G1 (e-mail: cclark@mecheng1.uwaterloo.ca).

Jan P. Huissoon is a Professor at the Dept. of Mechanical Engineering, University of Waterloo, ON, Canada N2L 3G1 (e-mail: jph@uwaterloo.ca). level navigation systems with a high level DGPS/DR sensor integration system and a map database. Their system is able to detect which lane the car is driving in and when a car is changing lanes. Another approach [4] integrates an Inertial Measurement Unit (IMU) with the GPS receiver to allow for accurate vehicle positioning. It also uses Real-Time Kinematic DGPS (RTK-DGPS) receiver supported by DGPS base stations at the test site and a lane-level-detailed digital map. Other research directions can also be found in [5][6][7][8][9].

Most of these systems usually have to involve in complicated image processing algorithms and/or costly equipments such as highly accurate sensors, high performance computers, etc. With that in mind, we approach the problem by using sharing information between vehicles to determine their lane positions. Inter-vehicle communication and co-operative driving systems have been in development for a while [10][11][12][13]. With the availability of GPS systems, it is practical to locate a vehicle within certain accuracy. However, GPS data are usually off the road and do not provide the exact position of vehicles due to degradation or multi-path problem. Therefore, it is challenging to determine the exact lanes that vehicles are traveling in even when the digital map of the road network is available.

In this paper, the authors describe a recent research effort in the area of low-cost lane-level position determination that can support a large number of transportation applications. A Markov-based approach which computes the lane position of transporting vehicles such as: cars, buses, etc, is proposed. Our fundamental assumption is the availability of a number of vehicles equipped with GPS receivers. Each vehicle has an ability to communicate with other vehicles within a certain radius and be able to send its information regarding position to other vehicles via ad-hoc network. In our simulations and experiments, the lane-level positioning algorithm works well when the vehicles are even $200 \mathrm{~m}$ away from each other.

This paper is organized as follows: Section II gives short introduction to Global Positioning Systems (GPS) and the architecture of the lane positioning system. Section III deals with the Markov localization algorithm in detail and its specific application in lane positioning problem. The simulation and experimental results are discussed in Section IV and V. In Section VI are some concluding remarks.

\section{GLOBAL POSITIONING SYSTEMS}

The GPS is the most convenient and accurate method for 
determining vehicle position in a global coordinate system. It does this by using satellites and receivers. There are currently 24 satellites in orbit operated by the US Department of Defense that provide worldwide coverage 24 hours a day, 7 days a week, in all weather. A detailed but concise description of GPS is provided in [14]. How the system works is by the satellites sending information to receivers. This information includes time, $(x, y, z)$ position, and satellites strength among other things. The receivers pick up information from at least four satellites and use this to determine the users' $(x, y, z)$ location according to various measurements of the pseudo-range between the satellite and the receiver antenna. The standard deviation of a standard GPS is on the order of $10-20 \mathrm{~m}$ and the GPS measurements for a car are usually off the road. Therefore, determine which lane a vehicle is traveling in is difficult if only GPS data from one vehicle are available. In this paper, it will be shown that by using sharing GPS data between vehicles that are traveling close to each other, the lane-level position for each vehicle can be determined. The architecture for the system is shown in Fig. 1. Each vehicle is equipped with a GPS receiver and a computer to implement lane positioning algorithm and communicate with other vehicles.
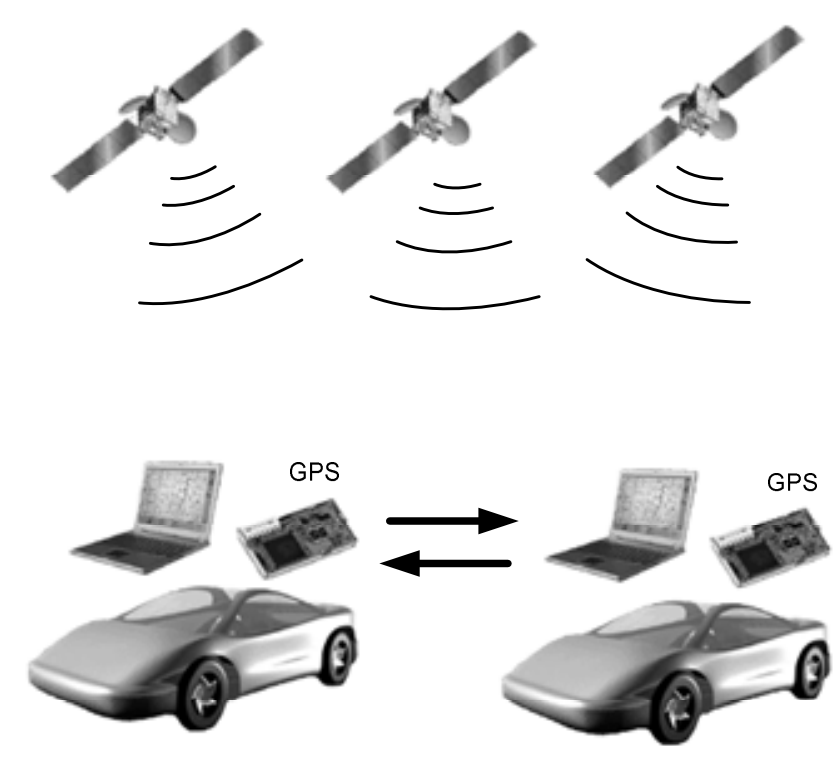

Fig. 1. Architecture for lane positioning system.

\section{MARKOV LOCALIZATION}

Markov localization addresses the problem of state estimation from sensor data. Markov localization is a probabilistic algorithm: instead of maintaining a single hypothesis as to which lane the vehicles might be, Markov localization maintains a probability distribution over the space of all such hypotheses. The probabilistic representation allows it to weight these difference hypotheses in a mathematically sound way.

To introduce the major concepts, let us begin with a simple case, followed by a mathematical derivation of the algorithm. The readers may notice that Markov localization is a special case of probabilistic state estimation [15][16][17].

Let us start with the simplest case: two vehicles traveling on a two-lane road. The two vehicles are assumed close enough to be able to communicate with each other. The GPS data provided by the GPS receiver are usually off the road and not accurate enough to give correct lane position for each vehicle. The target is to determine which vehicle is travel in which lane. As usual in Markov localization approach, call $\hat{P}_{t}\left(v_{1}=l_{a}, v_{2}=l_{b}\right)$ the probability that vehicle 1 is traveling in lane $a$ and vehicle 2 is in lane $b$ at time $t$; where $a$ and $b$ are either 1 or 2 . Therefore there are totally four possibilities, which are $\hat{P}\left(v_{1}=l_{1}, v_{2}=l_{1}\right)$, $\hat{P}\left(v_{1}=l_{1}, v_{2}=l_{2}\right), \quad \hat{P}\left(v_{1}=l_{2}, v_{2}=l_{1}\right), \quad$ and $\hat{P}\left(v_{1}=l_{2}, v_{2}=l_{2}\right)$. Initially, all the probabilities of where the vehicles might be have an equal value of 0.25 . In the course of the vehicles' mission (i.e., when $t>0), \hat{P}$ is updated through two basic steps: (1) prediction, and (2) correction.

\section{A. Prediction:}

In the prediction step, the state of a vehicle is modeled through the conditional probability $P\left(v_{i}=l_{a} \mid v_{i}=l_{j}\right)$, which denotes the probability for a motion action that carries vehicle $i$ from lane $j$ to lane $a$. When the vehicle moves, $P\left(v_{i}=l_{a} \mid v_{i}=l_{j}\right)$, which models the uncertainty in the vehicle's dynamics, is used to compute the probability distribution at time $t$ as

$$
P_{t}\left(v_{i}=l_{a}\right) \leftarrow \sum_{j=1}^{2} P_{t-1}\left(v_{i}=l_{a} \mid v_{i}=l_{j}\right) P_{t-1}\left(v_{i}=l_{j}\right),
$$

where $P\left(v_{i}=l_{a}\right)$ is the probability that vehicle $i$ is traveling in lane $a$. This step is repeated for both vehicles and then takes the multiplication to form the combined probabilities:

$$
\hat{P}_{t}\left(v_{1}=l_{a}, v_{2}=l_{b}\right)=P_{t}\left(v_{1}=l_{a}\right) P_{t}\left(v_{2}=l_{b}\right) .
$$

The conditional probabilities $P_{t}\left(v_{i}=l_{a} \mid v_{i}=l_{j}\right)$ for a vehicle are computed by comparing its current GPS position to the position at the last time step $t-1$. For example, by calculating the distance $\Delta D$ from the GPS reading at time $t$ to the vehicle's heading direction (obtained from two successive GPS readings) in the last time step, the probability at which vehicle 1 switches to lane 2 (see Fig. 2) can be computed. If $\Delta D$ is larger than the lane width, then the probability that vehicle 1 switches to lane 2 is close to $100 \%$. In a similar fashion, one can come up with all the conditional probabilities $P_{t}\left(v_{i}=l_{a} \mid v_{i}=l_{j}\right)$ that cover all possible situations. 


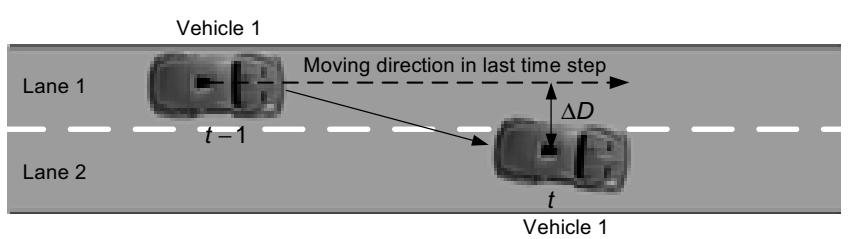

Fig. 2. Prediction step: this figure illustrates a vehicle switching lane and its position is unknown. The distance $\Delta D$ from the current GPS reading to the estimated heading direction of the vehicles in the last time step is used to calculate the conditional probability.

Since it is necessary to calculate $\Delta D$ of a vehicle between two successive time steps, a curve in the road could cause a problem where it is unclear as to whether the vehicle is switching lanes, or just following the curve in the road. For example Fig. 3 shows that the vehicle is still in lane 1, but a large value of $\Delta D$ due to the curve would falsely indicate that the vehicle is switching to lane 2 .

To resolve this discrepancy, the lane finding algorithm must estimate the radius of the lane curvature and compensate the drift caused by the curve. This can be done by using the least square method based on successive GPS readings. In our simulations and experiments five successive GPS readings were used to estimate this curvature.

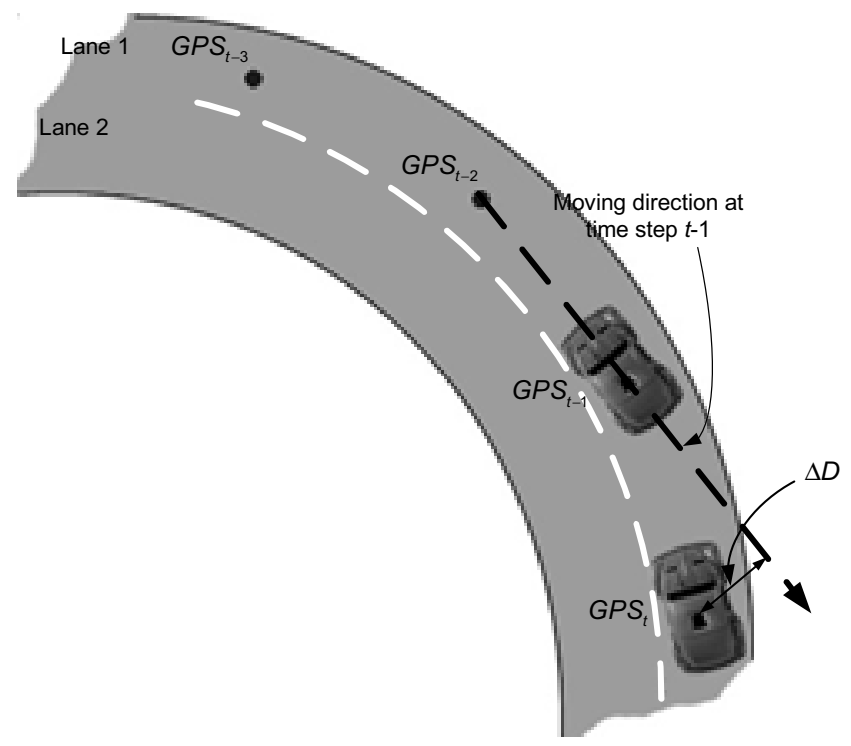

Fig. 3. Radius of curvature estimation: this figure illustrates the GPS readings for a vehicle assumed to be traveling in lane 1 . The position and moving direction of the vehicle are estimated from GPS data. The GPS measurements are not necessarily exactly in lane 1 and the actual vehicle does not necessarily overlay with the GPS measurements as the figure shows.

\section{B. Correction:}

Denote $z$ the GPS measurements, that come in at time step $t$, for both vehicles and $P\left(z \mid v_{1}=l_{a}, v_{2}=l_{b}\right)$ the probability of perceiving $z$ when the two vehicles are in lanes $a$ and $b$, respectively. When the GPS measurements are taken into account, $P\left(z \mid v_{1}=l_{a}, v_{2}=l_{b}\right)$ is used to update the probability distribution at time $t$ using Bayes' rule

$$
P_{t}\left(v_{1}=l_{a}, v_{2}=l_{b} \mid z\right) \leftarrow \frac{P_{t}\left(z \mid v_{1}=l_{a}, v_{2}=l_{b}\right) \hat{P}_{t}\left(v_{1}=l_{a}, v_{2}=l_{b}\right)}{P_{t}(z)},
$$

where $P(z)$ has the purpose of normalizing the sum of all $P_{t}\left(v_{1}=l_{a}, v_{2}=l_{b} \mid z\right)$.

We will show how $P\left(z \mid v_{1}=l_{a}, v_{2}=l_{b}\right)$ is provided using the sharing of GPS data between vehicles.

Suppose that at time $t$ new GPS data $z$ are available consisting of the positions of the two vehicles in the form of their $x$ and $y$ coordinates. From that, the distance, which is $z$, between the two vehicles with respect to their moving directions (see Fig. 4) is calculated.

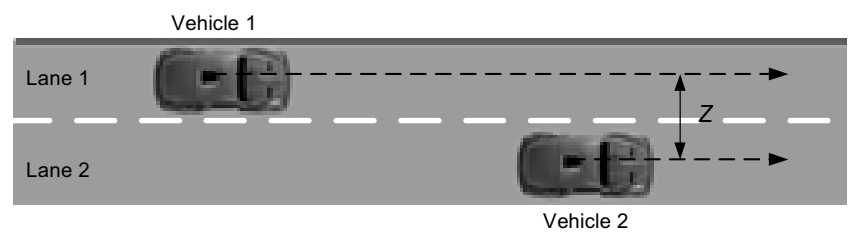

Fig. 4. Correction step: this figure illustrates two vehicles traveling on a highway. The distance $z$ is used to update the belief in prediction step.

The result for two-vehicle-and-two-lane-road can be extended to the general case. Denote the number of vehicles that are communicating with each other and the number of lanes by $n_{v}$ and $n_{l}$ respectively. Let $\hat{P}_{t}\left(v_{1}=l_{a}, v_{2}=l_{b}, \ldots, v_{n_{v}}=l_{x}\right)$ be the probability that vehicle 1 is traveling in lane $a$, vehicle 2 is in lane $b$, etc., at time $t$ where $a, b, \ldots, x$ vary from 1 to $n_{l}$. The probability distribution at time $t$ for the prediction step is given by

$$
P_{t}\left(v_{i}=l_{a}\right) \leftarrow \sum_{j=1}^{n_{t}} P_{t-1}\left(v_{i}=l_{a} \mid v_{i}=l_{j}\right) P_{t-1}\left(v_{i}=l_{j}\right),
$$

and

$$
\begin{aligned}
& \hat{P}_{t}\left(v_{1}=l_{a}, v_{2}=l_{b}, \ldots, v_{n_{v}}=l_{x}\right)= \\
& \quad=P_{t}\left(v_{1}=l_{a}\right) P_{t}\left(v_{2}=l_{b}\right) \ldots P_{t}\left(v_{n_{v}}=l_{x}\right) .
\end{aligned}
$$

Bayes' rule for the correction step is

$$
\begin{aligned}
& P_{t}\left(v_{1}=l_{a}, v_{2}=l_{b}, \ldots, v_{n_{v}}=l_{x} \mid z\right) \leftarrow \\
& \quad \leftarrow \frac{P_{t}\left(z \mid v_{1}=l_{a}, v_{2}=l_{b}, \ldots, v_{n_{v}}=l_{x}\right) \hat{P}_{t}\left(v_{1}=l_{a}, v_{2}=l_{b}, \ldots, v_{n_{v}}=l_{x}\right)}{P_{t}(z)} .
\end{aligned}
$$

With the developed Markov-based algorithm, we are able to implement simulations and experiments which will be discussed in the next Sections.

\section{SimULATION RESUlts}

To implement simulations, the software VISSIM [18] was used. VISSIM is a microscopic, time step and behavior based simulator developed to analyze the full range of functionally classified roadways. It is capable of modeling 
traffic with various control measures in 3D environment. VISSIM lets us communicate and control the behaviors of vehicle through a dynamic link library (DLL) file complied from $\mathrm{C} / \mathrm{C}++$ code. Vehicle parameters from the external driver model DLL output function are stored within member variables of a designated vehicle class object.

For a precise analysis of the algorithm, a 3D map of a road based on an actual highway, as shown in Fig. 5, was built in VISSIM and a number of simulations with different situations were implemented. In all simulations, the GPS data are modeled by adding some noise to the positions of vehicles.

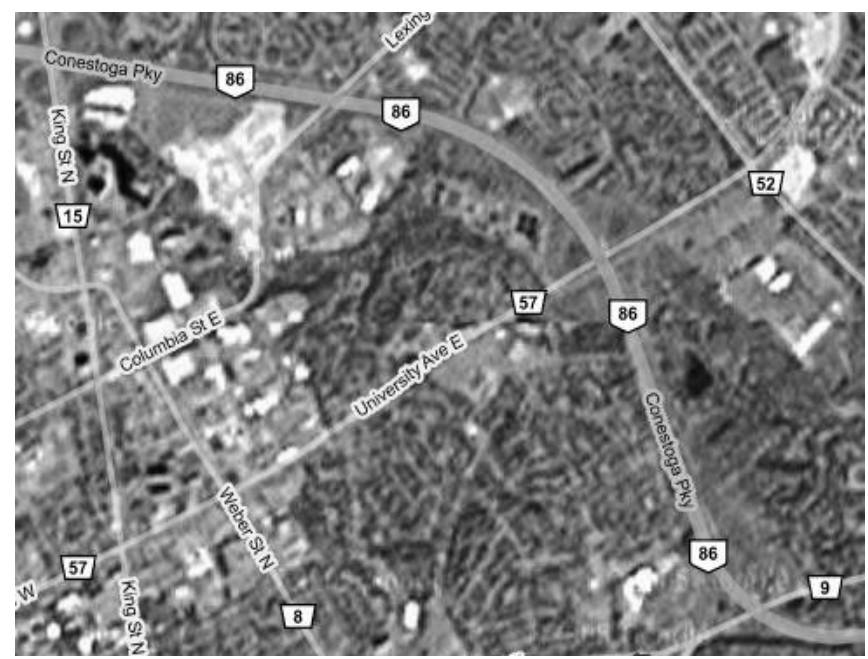

Fig. 5. Highway 86, Waterloo, ON, Canada.

Fig. 6 and Fig. 7 show a simulation with three cars and a two-lane road. The scenario is as follows: Initially, car 1 is in lane 1, car 2 and car 3 are both in lane 2. After 6 seconds, car 2 switches to lane 1 . Car 1 switches to lane 2 after 16 seconds and car 3 moves to lane 1 after 26 seconds (Fig. 7). One can see that the algorithm accurately estimates the lane positions of vehicles. The corresponding probabilities are given in Fig. 6. In practice, as the number of vehicles grows larger, the algorithm will be able to estimate the lane positions faster and more reliable.

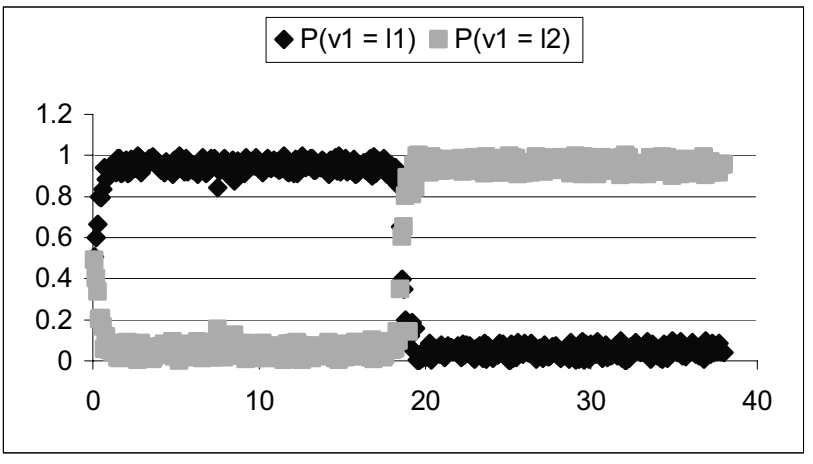

(a)

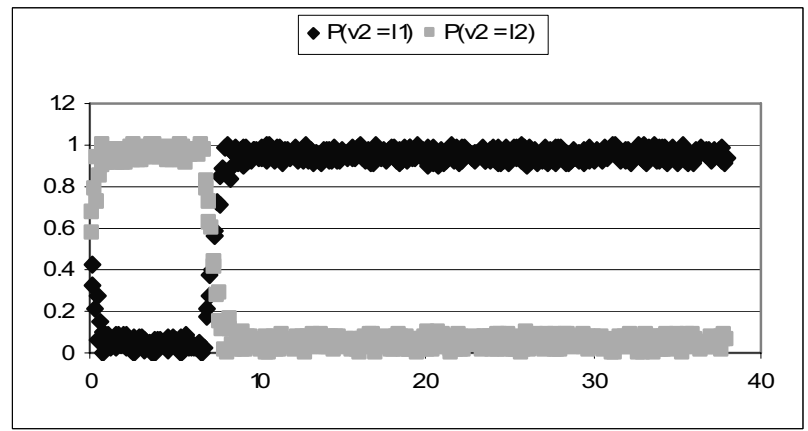

(b)

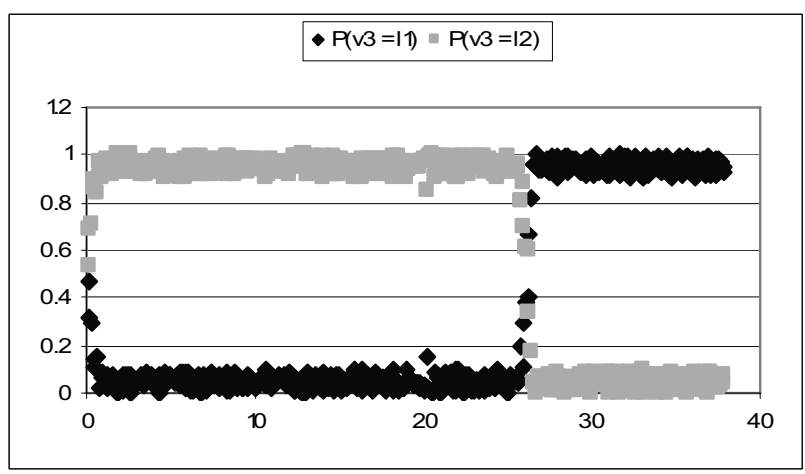

(c)

Fig. 6. Probability distribution for simulation of three cars (car 1 to car 3 from top to bottom) on a two-lane highway.
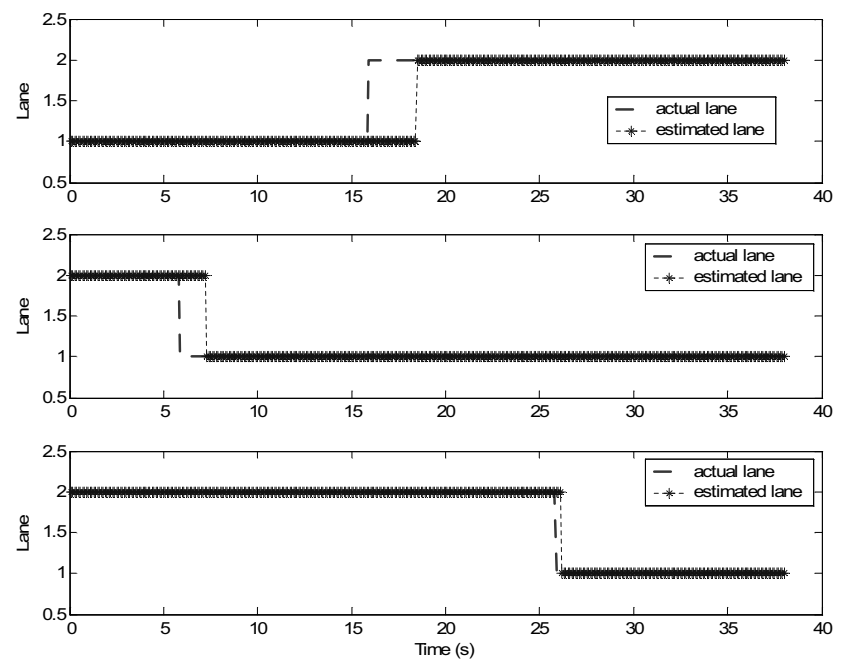

Fig. 7. Simulation result for three cars (car 1 to car 3 from top to bottom) on a two-lane highway.

\section{EXPERIMENTAL RESULTS}

To validate the algorithm, a number of experiments with different scenarios were implemented on the same highway. The real GPS data were also collected for the cars used in the test.

One of those experiments is shown in Fig. 8 and Fig. 9. In this experiment, two cars equipped with low cost GPS receivers with different sampling rates and variances 
maneuvered on the highway. The GPS receiver for the first car is Garmin 18 and has the sampling rate of $5 \mathrm{~Hz}$. The GPS receiver for the second car is LocSense 40-CM whose sampling rate is $1 \mathrm{~Hz}$. Both GPS receivers output National Marine Electronics Association (NMEA) 0183 standard messages. The first GPS receiver has much lower noise compared to the second one. This is reflected in Fig. 8 and Fig. 9. One can see that the plots for car 2 are much noisier than that of the first car.

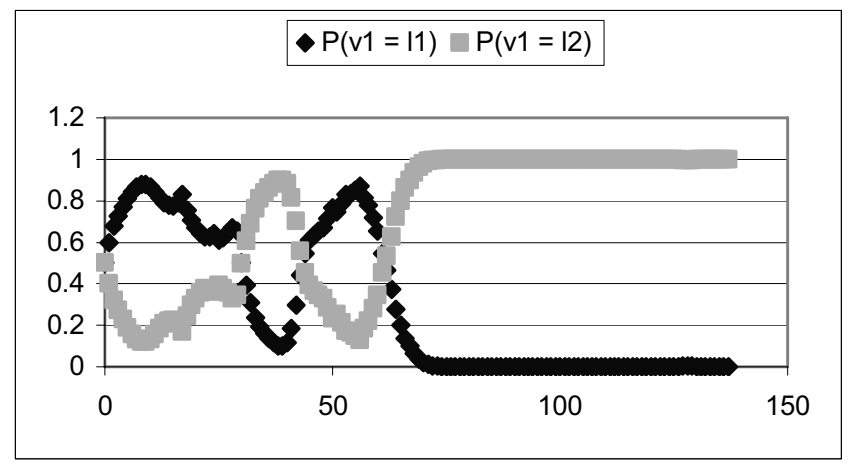

(a)

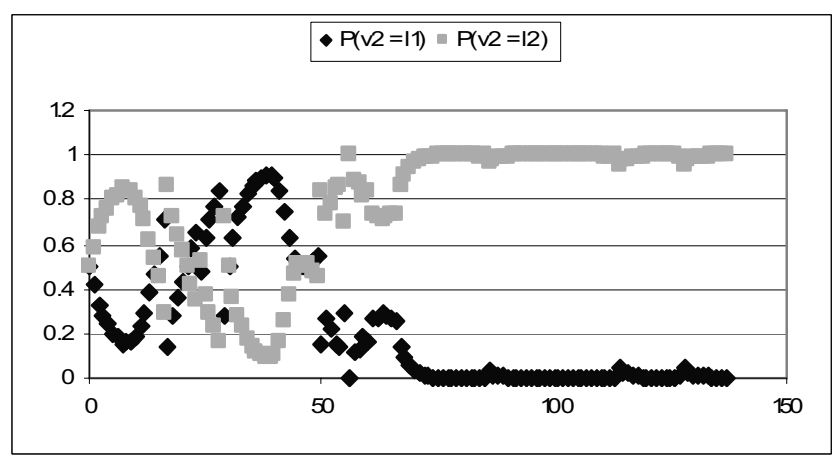

(b)

Fig. 8. Probability distribution for experiment.
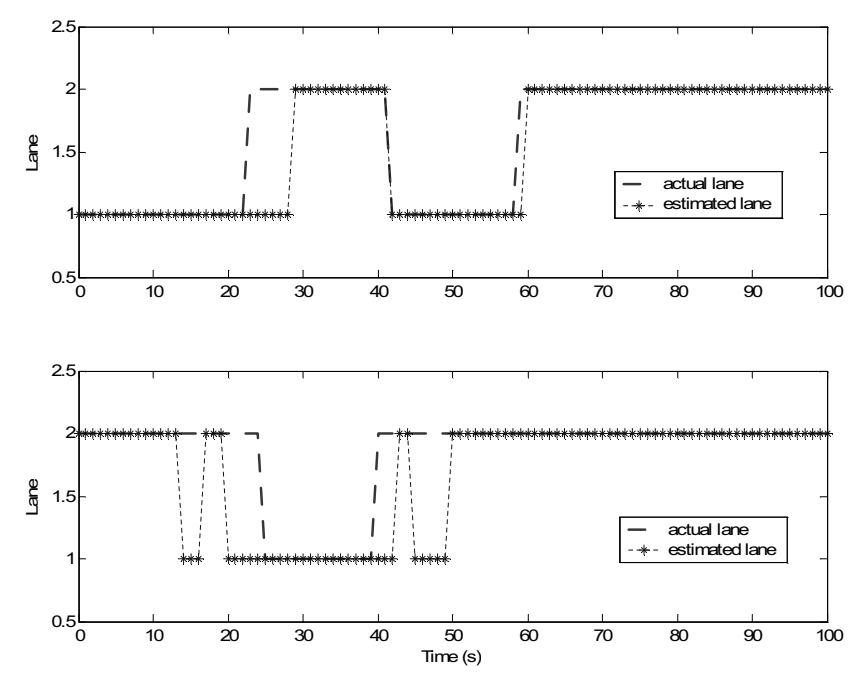

Fig. 9. Lane estimation result for two cars on highway (car 1 to car 2 from top to bottom).
To refine the estimation result, the GPS data for car 2 were let run though a particle filter [19] fused with a Butterworth filter (one can use other types of filters instead) to filter out the unwanted measurement noise (see Fig. 10). Due to the limited space, the detailed discussion about the used particle filter will be reserved for a future report. Fig. 11 and Fig. 12 show improvements compared to the results shown in Fig. 8 and Fig. 9. This improvement indicates that one can fuse low cost GPS receivers with a filter to obtain low-noised GPS measurements instead of using expensive sensors.

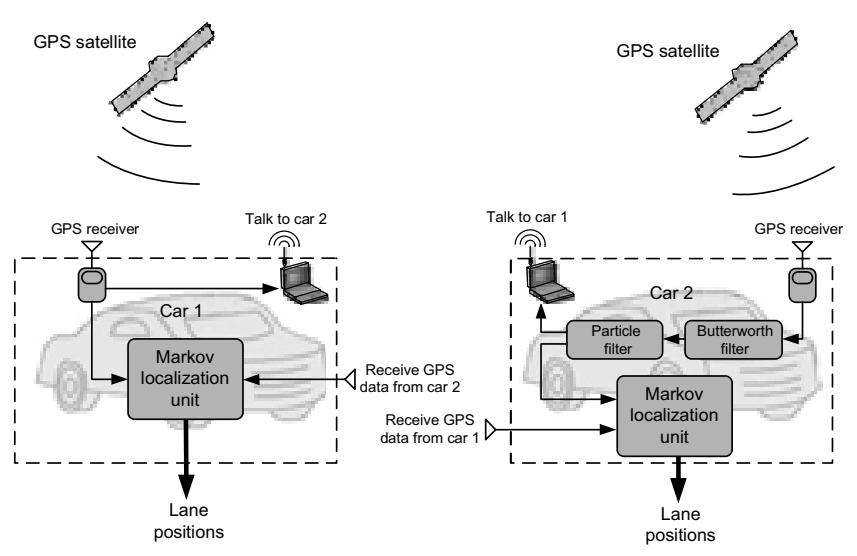

Fig. 10. Co-operative lane positioning diagram with filters.

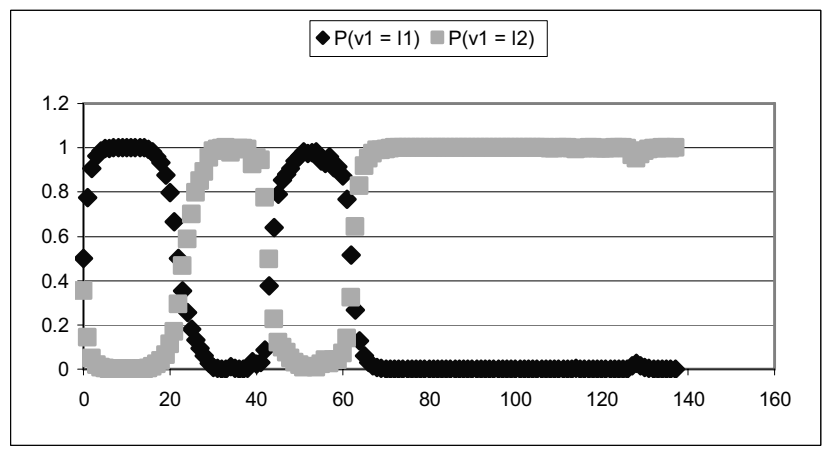

(a)

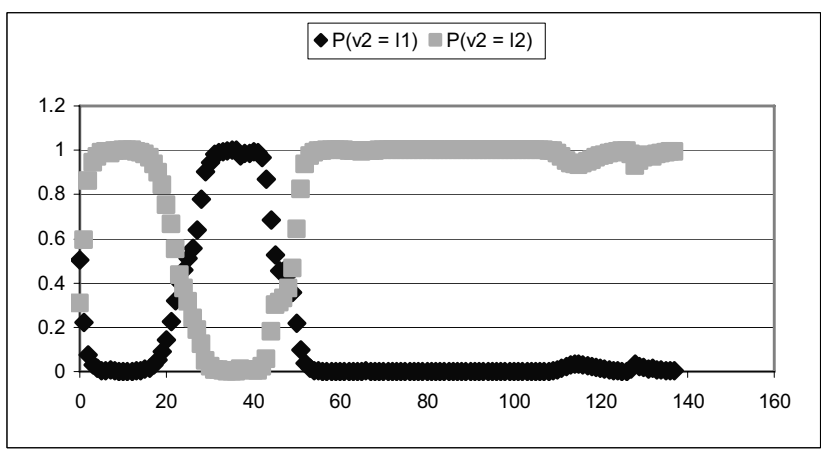

(b)

Fig. 11. Improved probability distribution for experiment. 

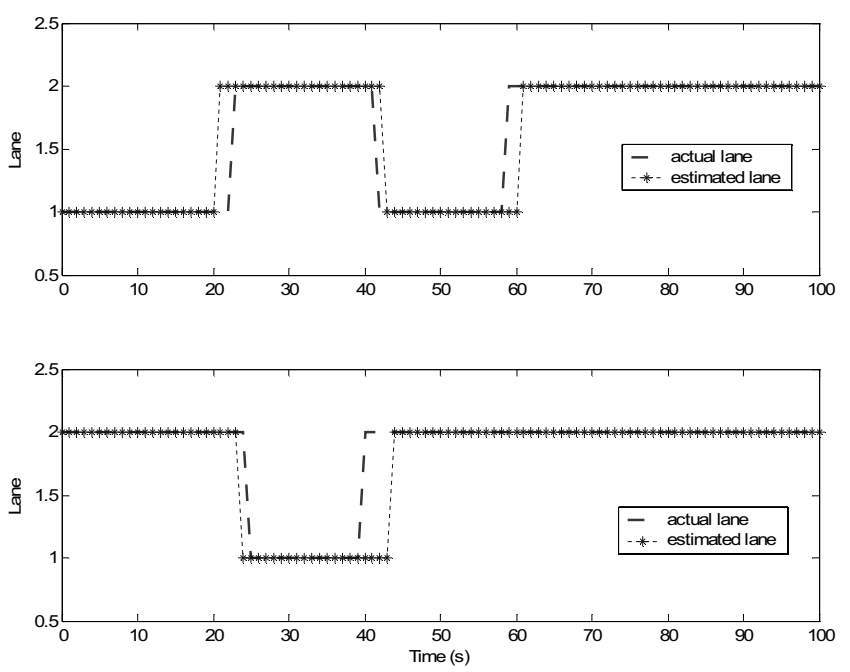

Fig. 12. Improved results with particle filtering.

\section{CONCLUSION}

This paper has proposed a new lane position estimation algorithm that uses a Markov-based approach based on cooperative driving models.

Compared to conventional lane positioning methods which usually deal with complicated image processing techniques and/or expensive equipments, only low cost GPS receivers and a simple localization algorithm are used. Simulation and experiment results have proved the efficiency of the algorithm, even when the GPS data are highly degraded.

Future research will continue with more complicated situations such as determining lane position on highways with intersections. Besides the lane models, such problem requires a per-lane model of intersection behavior. For example, the positioning system may indicate that $100 \%$ of drivers in the left lane at a particular intersection turn left, $50 \%$ of drivers in the right lane go straight, and $50 \%$ turn right.

It is hoped that the knowledge acquired from this research will lead to the future development of actual lane positioning systems.

\section{ACKNOWLEDGMENT}

The authors would like to thank AUTO21, Canada, for financially supporting this research.

\section{REFERENCES}

[1] J. Du, J. Masters and M. Barth, "Lane-level positioning for in-vehicle navigation and automated vehicle location (AVL) systems", in Proc. Of IEEE Intelligent Transportation Systems Conference, Washington, USA, 2004, pp. 35-40.

[2] S. S. Ieng, J. Vrignon, D. Gruyer and D. Aubert, "A new multi-lane detection using multi-camera for robust vehicle localtion", in Proc. of IEEE Intelligent Vehicles Symposium, Nevada, USA, 2005, pp. 700705.

[3] G. Pierre-Yves and K. Jeff, "Enhanced navigation system for road telematics", 3rd Swiss Transportation Research Conference, 2003, pp. 1-16.
[4] Enhanced digital mapping project final report, U.S. Department of transportation, Washington, 2004.

[5] K. Y. Chiu and S. F. Lin, "Lane detection using color-based segmentation", in Proc. Of the IEEE Intelligent Vehicle Symposium, 2005, pp. 706-711.

[6] B. D. Stewart, I. Reading, M. S. Thomson, T. D. Binnie, K. W. Dickinson and C. L. Wan, "Adaptive lane finding in road traffic image analysis", in Proc. of the 7th IEEE Int. Conference on Road Traffic Monitoring and Control, London, UK, 1994, pp. 133-136.

[7] J. Gangyi, C. Yanhua, Y. Mei and Z. Yi, "Approach to lane departure detection", in Proc. of IEEE 5th Int. Conference on Signal Processing, ICSP'2000, pp. 971-974.

[8] A. H. S. Lai and N. H. C. Yung, "Lane detection by orientation and length discrimination", IEEE Trans. On Systems, Man, and Cybernetics-Part B: Cybernetics, vol. 30, no. 4, August 2000, pp. 539-548.

[9] T. K. Lim and M. A. Do, "Camera detection, classification, and positioning of vehicles on a multi-lane road", in Proc. of IEEE Singapore International Conference on Information Engineering, vol. 1, Singapore, 1993, pp. 374-378.

[10] M. M. Artimy, W. Robertson and W. J. Phillips, "Connectivity in intervehicle ad-hoc networks", Engineering Canadian Conference on Electrical and Computer, vol. 1, May 2004, pp. 293-298.

[11] J. Blum, A. Eskandarian and L. Hoffman, "Performance characteristics of inter-vehicle ad hoc networks", in Proc. Of the IEEE 6th International Conference on Intelligent Transportation Systems, China, 2003, pp. 114-119.

[12] Z. D. Chen, H. T. Kung and D. Vlah, "Ad hoc relay wireless networks over moving vehicles on highways", in Proc. Of the 2nd ACM International Symposium on Mobile Ad Hoc Networking and Computing, 2001.

[13] W. Enkelmann, "FleetNet applications for inter-vehicle communication", in Proc. Of the IEEE Intelligent Vehicles Symposium, Ohio, USA, 2003, pp. 162-167.

[14] J. Farrel and M. Barth, The Global Positioning System and Inertial Navigation: Theory and Practice, McGraw-Hill, 1999.

[15] S. J. Russell and P. Norvig, Artificial Intelligence: A Modern Approach, Prentice Hall, 1995.

[16] D. Fox, Markov Localization: A Probabilistic Framework for Mobile Robot Localization and Naviagation, PhD thesis, Dept. of Computer Science, University of Bonn, Germany, December 1998.

[17] S. Koenig and R. Simmons, "A robot navigation architecture based on partially observable markov decision process models", In Kortenkamp et al. AI-ROBOTS.

[18] VISSIM

[Online].

Available: http://www.trafficgroup.com/services/vissim.html

[19] I. M. Rekleitis, "A particle filter tutorial for mobile robot localization TR-CIM-04-02", Centre for Intelligent Machines, McGill University, Canada. 